\title{
Isogenic-induced endothelial cells enhance osteogenic differentiation of mesenchymal stem cells on silk fibroin scaffold
}

\author{
Sindhuja D Eswaramoorthy' ${ }^{1}$ Nandini Dhiman ${ }^{1}(\mathbb{D})$, Gayathri Korra ${ }^{2}$, Carlo M Oranges ${ }^{3}$, Dirk \\ J Schaefer ${ }^{3}$, Subha N Rath**, ${ }^{*}, 1$ \& Srinivas Madduri*, $\neq, 3,4,5$ \\ ${ }^{1}$ Department of Biomedical Engineering, Indian Institute of Technology Hyderabad, 502285 Telangana, India \\ ${ }^{2}$ Sri Sai Krishna Multi Specialty Hospital, Department of Obstetrics and Gynecology, Sangareddy 502001, Medak, Telangana, India \\ ${ }^{3}$ Department of Plastic, Reconstructive, Aesthetic \& Hand Surgery, University Hospital Basel, University of Basel, Basel, Switzerland \\ ${ }^{4}$ Department of Biomedicine, University of Basel, $\mathrm{CH}-4031$ Basel, Switzerland \\ ${ }^{5}$ Department of Biomedical Engineering, University of Basel, $\mathrm{CH}-4123$ Allschwil, Switzerland \\ *Author for correspondence: Tel.: +41 061556 5049; Fax: +41 061207 5409; srinivas.madduri@usb.ch \\ **Author for correspondence: Tel.: +91 402301 7111; Fax: +91 402301 6032; subharath@iith.ac.in \\ $\ddagger$ Authors contributed equally
}

\begin{abstract}
Aim: We investigated the role of induced endothelial cells (iECs) in mesenchymal stem cells (MSCs)/iECs coculture and assessed their osteogenic ability on silk fibroin nanofiber scaffolds. Methods: The osteogenic differentiation was assessed by the ALP assay, calcium assay and gene expression studies. Results: The osteogenic differentiation of the iECs co-cultures was found to be higher than the MSCs group and proximal to endothelial cells (ECs) co-cultures. Furthermore, the usage of isogenic iECs for co-culture increased the osteogenic and endothelial gene expression. Conclusion: These findings suggest that iECs mimic endothelial cells when co-cultured with MSCs and that one MSCs source can be used to give rise to both MSCs and $\mathrm{iECs}$. The isogenic MSCs/iECs co-culture provides a new option for bone tissue engineering applications.
\end{abstract}

First draft submitted: 19 December 2018; Accepted for publication: 11 June 2019; Published online: 26 July 2019

Keywords: co-culture $\bullet$ induced endothelial cells $\bullet$ isogenic source $\bullet$ osteogenic differentiation $\bullet$ silk fibroin nanofiber - umbilical cord-derived mesenchymal stem cells

\section{Background}

Mesenchymal stem cells (MSCs) can self renew, proliferate and differentiate into multiple lineages. This makes them a promising candidate for cell-based therapies in tissue engineering. They can be isolated from different sources and differentiated into several lineages under suitable induction conditions, especially to osteogenic, adipogenic, myogenic and chondrogenic differentiation [1].

The umbilical cord is an attractive source for the isolation of MSCs because of abundance, fewer ethical concerns unlike embryonic stem cells, and ease of culture [2]. Umbilical cord MSCs (UCMSCs) propose to be a promising source of MSCs that can be thawed, expanded and stored in cell banks for therapeutic applications at the time of need [3].

Enhanced osteogenic differentiation can be achieved by various methods such as stimulation using chemical supplements [4,5], electrical stimulation [6,7], bioreactor stimulation [8,9] and co-culture with endothelial cells (ECs) [10,11]. Use of MSCs-ECs co-culture, with osteogenic supplements, is an effective tool to achieve osteogenic differentiation. It is known that the presence of ECs with MSCs has a profound effect on their proliferation, differentiation, cell fate, and their resultant angiogenesis and osteogenesis [4]. Several reports have suggested co-culturing of MSCs and ECs together for enhanced osteogenic differentiation as compared with MSC monocultures [5-7]. Meinel et al. reported enhanced bone formation potential when MSCs were predifferentiated toward osteoblastic lineage [8]. Binder and group also reported increased cell survival when cells were precultured in osteogenic medium [9]. There are also reports suggesting that bone morphogenetic protein-2 synthesized by ECs increases the osteogenic differentiation in co-culture [12]. However, the ECs isolated from large blood vessels have low prolifera-

Future Medicine 
tion rate and are likely to de-differentiate in culture after prolonged expansion [10,11]. Also, autologous isolation of these ECs requires sacrificing a vessel of the patient [13]. In addition, getting both the cell types (MSCs and ECs) from autologous source is much challenging than any single cell type.

To overcome these limitations and to avoid the ethical concerns of using two different cell types, the ability of MSCs to differentiate into endothelial phenotype is investigated. In the initial phase of vascular development, ECs in the embryo are obtained through in situ differentiation of the multipotent mesodermal progenitor cells [14,15]. Corotchi $e t$ al. proved that culture conditions and isolation procedures could lead to various cell lineages, starting with the same MSCs derived from Wharton's jelly. They also reported a cell population that co-expressed mesenchymal and EC markers [16]. MSCs have been reported to differentiate into endothelial lineage by many other groups as well [17-19]. These particular kind of cells obtained by endothelial differentiation of MSCs are induced ECs (iECs). iECs were found to stimulate in vitro differentiation of bone marrow MSCs (BM-MSCs) and stimulate osteogenesis in vivo by direct contact with BM-MSCs [20]. It was also reported that BM-MSCs offered new choices for engineering of artificial tissues based on autologous MSCs and vascularized engineered tissues [21].

The therapeutic potential of the MSCs-ECs co-culture has been hampered due to lack of appropriate structural scaffolds supporting these cell types. Hence, silk-based scaffolds have been selected for this study. The application of silk-based biomaterials has been proved to be beneficial for bone regeneration because of their low inflammatory potential, excellent biocompatibility and ability to degrade in vivo entirely within 2-6 months [22]. Several studies have reported the use of electrospun silk fibroin (SF) nanofiber scaffolds (SFNS) in bone tissue engineering [16,23,24]. It has been proved that electrospun SFNS augment the osteogenic differentiation of MSCs and bone formation in bone defect models [25].

The rationale behind our study is to use one single cell source in a modified form for osteogenic MSC-EC co-culture system. Since UCMSCs have been shown to differentiate into the endothelial lineage [26], we have used these cells to obtain both the co-culture cell types, that is, MSCs and ECs. In this study, we intend to compare the osteogenic ability of iECs-based MSCs/iECs co-cultures with MSC monoculture and ECs-based MSCs/ECs co-culture. iECs can be used as a substitute for ECs for co-culture with MSC to induce osteogenic differentiation. Hence, cells from the same MSC source can be replaced in place of ECs, which need two separate cell sources paving the way for the use of autologous MSCs in co-cultures. The hypothesis of this study is twofold: first, that iECs can mimic the role of ECs in MSCs-iECs co-culture on SFNS; second, that MSCs-iECs co-culture can show enhanced osteogenic differentiation than MSC monoculture on SFNS.

\section{Methods}

\section{Isolation \& cell culture}

The umbilical cord was collected from the patient after informed consent. UCMSCs were obtained using tissue explant method [27]. Under sterile conditions, the cord was washed thoroughly with phosphate-buffered saline (PBS) to remove blood. With a sterile scalpel, the umbilical cord was cut into small pieces of about $5 \mathrm{~mm}^{3}$ and placed at an equal distance in a petri plate. The explants were left undisturbed for 5 min until attachment onto the plate. They were grown in DMEM containing 10\% fetal bovine serum, 2-mM L-glutamine and 1\% penicillinstreptomycin, and maintained at $37^{\circ} \mathrm{C}$ and $5 \% \mathrm{CO}_{2}$. The explants were left undisturbed for 4 days and media were changed twice weekly after that. After subculture, the medium was replaced with cytomix (Miltenyi Biotec GmbH, Germany). Human umbilical vein ECs (HUVECs) were purchased from Lonza (MD, USA) and grown in EC growth medium-2 (EGM-2, Promocell, Germany) at $37^{\circ} \mathrm{C}$ and $5 \% \mathrm{CO}_{2}$. PBS, DMEM and penicillinstreptomycin were purchased from Sigma-Aldrich (MO, USA); and L-glutamine and fetal bovine serum were purchased from Gibco (CA, USA).

\section{Endothelial differentiation of UCMSCs}

Endothelial differentiation was performed as described previously [10]. UCMSCs were seeded at a density of 10,000 cells $/ \mathrm{cm}^{2}$ and grown in EGM-2 (Promocell) for 12 days. The medium was changed twice weekly for iECs. Then, the iECs were co-cultured with MSCs and induced osteogenically in osteogenic medium containing 10-mM $\beta$-glycerophosphate (Sigma-Aldrich), $100 \mathrm{nM}$ dexamethasone (HiMedia, India), and 50- $\mu \mathrm{g} / \mathrm{ml} \mathrm{L}$-ascorbic acid (HiMedia). 


\section{Characterization of UCMSCs \& iECs}

UCMSCs demonstrated trilineage (osteogenic, adipogenic and chondrogenic) differentiation. As recommended by the International Society for Cellular Therapy, UCMSCs were analyzed for MSC markers such as CD73, CD90 and CD105, and non-MSC markers such as CD14, CD20, CD34 and CD45. Flow cytometry analysis was performed using Attune Acoustic Focusing Cytometer (Applied Biosystems, CA, USA) with the help of human MSC phenotyping kit (Miltenyi Biotec GmbH, Germany). iECs were assessed for their formation of tube-like structures on the basement membrane matrix preparation, Matrigel (NY, USA) as performed before [28]. They were also checked for lectin staining using Fluorescein labeled Lycopersicon Esculentum (Tomato) Lectin (Vector Laboratories, CA, USA).

\section{Preparation of regenerated silk fibroin solution}

As described earlier, SF was prepared using a modified protocol [29]. Briefly, cocoons from Bombyx mori were boiled in an aqueous solution of $0.02 \mathrm{M} \mathrm{Na}_{2} \mathrm{CO}_{3}$, rinsed with ultrapurified water (UPW) and dissolved in $9 \mathrm{M} \mathrm{LiBr}$ at $55^{\circ} \mathrm{C}$ to obtain a $10 \%(\mathrm{w} / \mathrm{v})$ solution. The solution was dialyzed (IL, USA; MWCO 3.500 Da) against UPW for $48 \mathrm{~h}$. After the desalination, a second dialysis against a polyethylene glycol 6000 solution (200 g/1.5 1 UPW) was performed to concentrate the SF solution up to $12.5 \%$. SF concentration was determined gravimetrically.

\section{Preparation of silk fibroin nanofiber scaffold by electrospinning}

Electrospinning was performed using previously described method [29]. The process was accommodated in a fume hood flushed with dry air to achieve a relative humidity of $22-28 \%$. SF was blended with poly(ethylene oxide) (PEO) of $900 \mathrm{kDa}$ to achieve stable, continuous spinning. PEO $(5 \%, \mathrm{w} / \mathrm{w})$ was dissolved in UPW by stirring for 5 days at room temperature, and the solution filtered through a $5 \mu \mathrm{m}$-pore size membrane filter. Then, $2 \mathrm{~g}$ of the PEO solution was mixed with $5 \mathrm{~g}$ of $12.5 \%$ SF solution by moderate stirring. The PEO-SF solution was electrospun at a flow rate of $0.8 \mathrm{ml} / \mathrm{h}$ using a syringe pump, a syringe with needle, a high voltage power supply (Fabrimex, Volketswil, Switzerland), and a voltage field of 10-14 kV between the needle and the grounded target placed at $19 \mathrm{~cm}$ from the needle. As target, a cover glass with 13-mm diameter was fixed onto a flat-grounded metal target of $5 \mathrm{~mm}$ diameter to produce nanofibers. The crystallinity of the SFNS was induced by water vapor treatment [29].

\section{Analysis of SF nanofiber}

Electrospun fibers were examined microscopically (Axiovert 35) and images were captured using Axio cam MRc5 (Carl Zeiss, Jena, Germany) digital camera for quantitative analysis of fiber homogeneous deposition. Fiber deposition and dimensions were analysed by using Image J software (NIH, MD, USA).

\section{Scanning electron microscopy}

Scanning electron microscopy (SEM) was used to determine the morphological appearance and diameter of the SF fibers. Water vapor-treated samples were platinum-coated prior to examination with a LEO 1530 GEMINI scanning electron microscope (Zeiss, Cambridge, UK). For the analysis of cell-seeded SFNS, composites were fixed with $3 \%$ glutaraldehyde.

\section{Experimental groups}

In this study, two cell types: UCMSCs and ECs were used primarily. Endothelial differentiation of UCMSCs for 12 days yielded iECs. ECs were not subject to any differentiation.

In our study, all the cultures were performed on SFNS, which were divided into three groups according to the cells used (Table 1). The monoculture MSC group was categorized as control, while iEC-based co-culture group was group B (MSC+iEC) and EC-based co-culture group was group C (MSC+EC). The samples were evaluated at predefined time points, 1 and 3 weeks. Only week-3 samples were considered for gene expression analysis.

Before seeding cells, the scaffolds were incubated overnight with PBS containing 5\% penicillin-streptomycin. After washing with PBS, scaffolds were exposed to ultra-violet light for $1 \mathrm{~h}$. Subsequently, the scaffolds were coated with $1 \mu \mathrm{g} / \mathrm{ml}$ of laminin (Sigma-Aldrich) and incubated at $37^{\circ} \mathrm{C}$ for $3 \mathrm{~h}$. The scaffolds were washed again with PBS before proceeding to cell seeding. For the control group, 20,000 MSCs per scaffold were seeded. For the other two co-culture groups, 10,000 cells of each cell type per scaffold were seeded. Cytomix was used as growth medium for the control group. For the co-culture groups (groups B and C), 1:1 cytomix-EGM-2 cocktail medium was used. 
Table 1. Experimental groups and study design involving mesenchymal stem cells, endothelial cells, induced endothelial cells and silk fibroin nanofiber scaffold.

\begin{tabular}{|c|c|c|c|c|c|c|}
\hline Groups & Cell 1 & Cell 2 & Live-dead assay (n) & AlamarBlue assay (n) & $\begin{array}{l}\text { ALP, Bicinchoninic } \\
\text { acid (BCA) and } \\
\text { calcium assay }(n)\end{array}$ & RT-qPCR (n) \\
\hline A (control) & MSC & - & 3 & 3 & 3 & 3 \\
\hline B & MSC & $\begin{array}{l}\text { iEC: endothelially } \\
\text { differentiated from } \\
\text { MSCs }\end{array}$ & 3 & 3 & 3 & 3 \\
\hline C & MSC & EC & 3 & 3 & 3 & 3 \\
\hline
\end{tabular}

After 2 days of culture on scaffolds, the medium was fully replaced with osteogenic medium for all groups. P4-P5 passage cells of all cell types were used for seeding on the silk nanofiber scaffold.

\section{Live-dead assay}

Fluorescein diacetate/propidium iodide (FDA/PI) assay was performed as discussed earlier [14]. The cell-scaffold composites were washed with PBS and stained with $2 \mu \mathrm{g} / \mathrm{ml}$ FDA (Molecular Probes, OR, USA). They were incubated at $37^{\circ} \mathrm{C}$ for $15 \mathrm{~min}$ and rinsed again with PBS. Then, the scaffolds were stained with $20 \mu \mathrm{g} / \mathrm{ml} \mathrm{PI}$ (Invitrogen, OR, USA) solution at room temperature for $2 \mathrm{~min}$. After rinsing with PBS, the scaffolds were immersed in PBS and viewed under a fluorescent microscope (Carl Zeiss Axio Observer Z1). Viable cells were stained with green color whereas dead cells stained red.

\section{AlamarBlue assay}

The cell-seeded SFNS were analyzed by AlamarBlue assay (Invitrogen) as described previously [14]. In short, 10\% AlamarBlue solution was added to the cell-scaffold composites and incubated at $37^{\circ} \mathrm{C}$ for $4 \mathrm{~h}$. The absorbance of the reduced dye was measured using a plate reader (Enspire multimode plate reader, Perkin Elmer, MA, USA) at 570 and $600 \mathrm{~nm}$. The percentage of dye reduction was calculated according to the manufacturer's protocol.

\section{Differentiation studies}

ALP staining \& assay

Staining was performed using ALP kit (Sigma-Aldrich) according to the manufacturer's protocol as discussed before [14]. In short, cells were fixed with the citrate fixative, and diazonium salt solution was used to stain the cells. The scaffolds were observed under phase contrast microscope (Olympus, Japan) and images were taken. The colored complex produced by the hydrolysis of p-nitrophenyl phosphate (Sigma-Aldrich) was measured at $460 \mathrm{~nm}$ absorbance by a microplate reader to assess the ALP content. The values were normalized to total protein, as measured by the following assay.

\section{$B C A$ assay}

The total protein content of the lysates was measured using BCA Protein Assay Kit (Pierce Biotechnology, IL, USA). The absorbance was measured at $540 \mathrm{~nm}$, as described by the manufacturer and reported before [28]. Total protein content values were used to normalize the ALP content of the lysates.

\section{Calcium assay \& staining}

In an alkaline buffer, calcium reacted with o-cresolphthalein, and the developed purple color was measured using a plate reader (Enspire multimode plate reader, Perkin Elmer) at a wavelength of $570 \mathrm{~nm}$. Calcium deposits were stained with Alizarin Red solution. Cells were fixed in 10\% formalin (Fisher Scientific, India), and 2\% Alizarin Red (HiMedia) was added to the scaffolds. The specimens were observed under phase contrast microscope (Olympus) and images were taken.

\section{Reverse transcription-quantitative PCR}

Osteogenic differentiation in the 3D silk scaffold co-culture for three groups was quantified by reverse transcriptionquantitative PCR (RT-qPCR) analysis. Total RNA from each sample was isolated using TRIzol reagent (Ambion, Life Technologies, CA, USA) and RNeasy Mini Kit (Qiagen, Hilden, Germany), as per manufacturer's protocol 


\begin{tabular}{|c|c|c|c|}
\hline S. No. & Gene (human) & Forward primer $\left(5^{\prime}-3^{\prime}\right)$ & Reverse primer $\left(5^{\prime}-3^{\prime}\right)$ \\
\hline 1. & $O C$ & AAAAAGGCATGAGGGGTCGT & CATGGTGTCGAGGAGCTTCA \\
\hline 3. & RPLP1 & CATTCTGCACGACGATGAGG & AACAAGCCAGGCCAAAAAGG \\
\hline
\end{tabular}

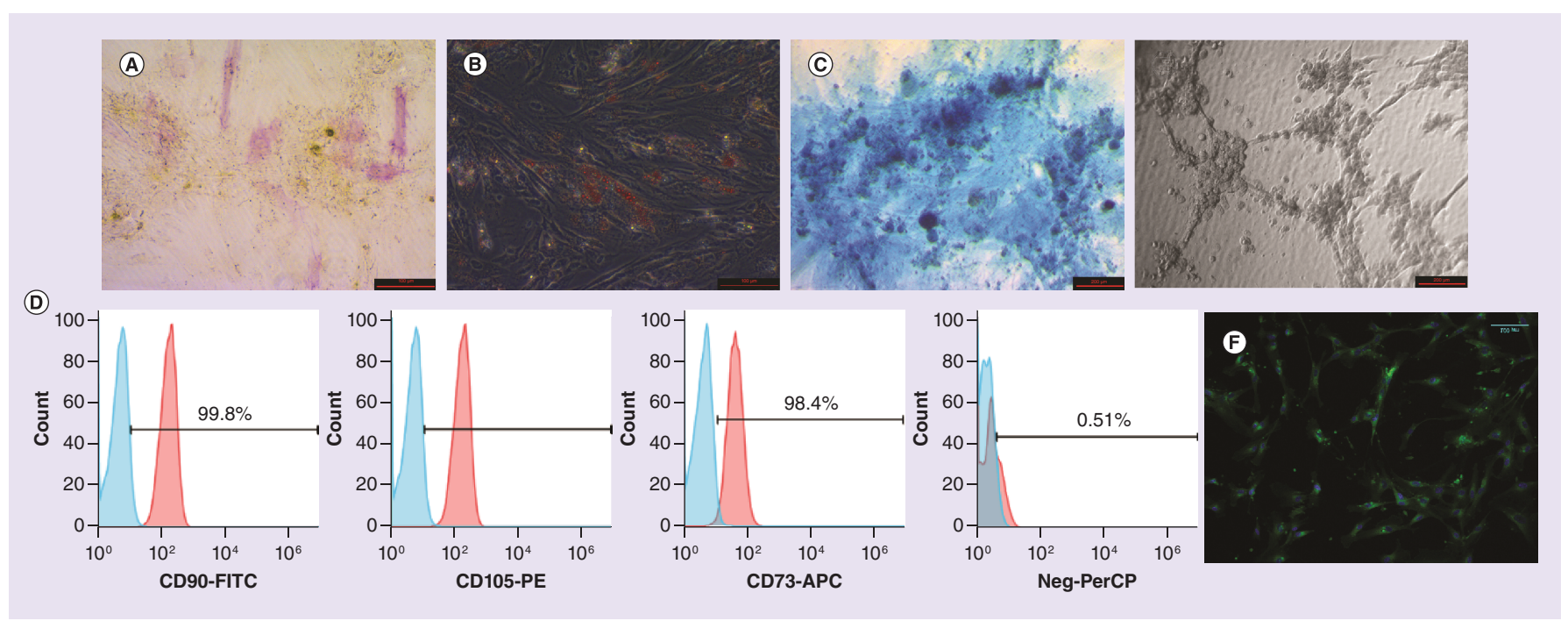

Figure 1. Characterization of umbilical cord mesenchymal stem cells. (A) ALP staining, (B) Oil Red O staining, (C) Alcian blue staining, (D) characterization of mesenchymal stem cells' marker proteins by FACS analysis, and characterization of induced endothelial cells: (E) Matrigel assay, and (F) lectin staining.

FACS: Fluorescence-activated cell sorting.

with a simple modification as described by our group previously [28]. After 3 weeks in culture, $500 \mu$ l of TRIzol were directly added to cell-laden silk scaffolds and homogenized using a pipette tip. The RNA concentration was determined by measuring absorbance at $260 \mathrm{~nm}$ using BioDrop Duo (UK).

A reverse transcription reaction was performed with $1 \mu \mathrm{g}$ pure total RNA using the QuantiTect Reverse Transcription Kit (Qiagen) according to the manufacturer's instructions. qPCR measurements of gene expression for osteogenic (OC: osteocalcin) and angiogenic marker (ANGPT1: angiopoietin1) were performed using a CFX96 Real-Time PCR Detection System (Bio-Rad Laboratories, CA, USA; Table 2). iTaq Universal SYBR Green Supermix (Bio-Rad Laboratories) was used for the reaction. The target gene expressions were normalized to that of the endogenous reference (RPLP1: Ribosomal Protein Lateral Stalk Subunit P1), and relative differences in target gene expression were determined using the $2^{-\Delta \Delta C_{T}}$ method. The normalized expression of each gene in co-cultured $3 \mathrm{D}$ silk scaffolds was expressed relative to the corresponding gene expression in control group A.

\section{Statistical analysis}

All statistical comparisons were performed by using one-way analysis of variance test followed by Bonferroni's post-hoc test (GraphPad Prism 7, CA, USA). For all analyses, a 95\% CI was used, and the results were considered significant at $\mathrm{p}<0.05$ level and $\mathrm{p}<0.01$.

\section{Results}

Characterization of UCMSCs \& iECs

UCMSCs were successfully differentiated into osteogenic, adipogenic and chondrogenic lineages (Figure 1A, B \& C). Flow cytometric analysis showed that UCMSCs intensively expressed MSC markers while lacking the hematopoietic and endothelial markers (Figure 1D). iECs produced the typical tube-like structures on Matrigel (Figure 1E). They also showed positive staining for lectin (Figure 1F). 


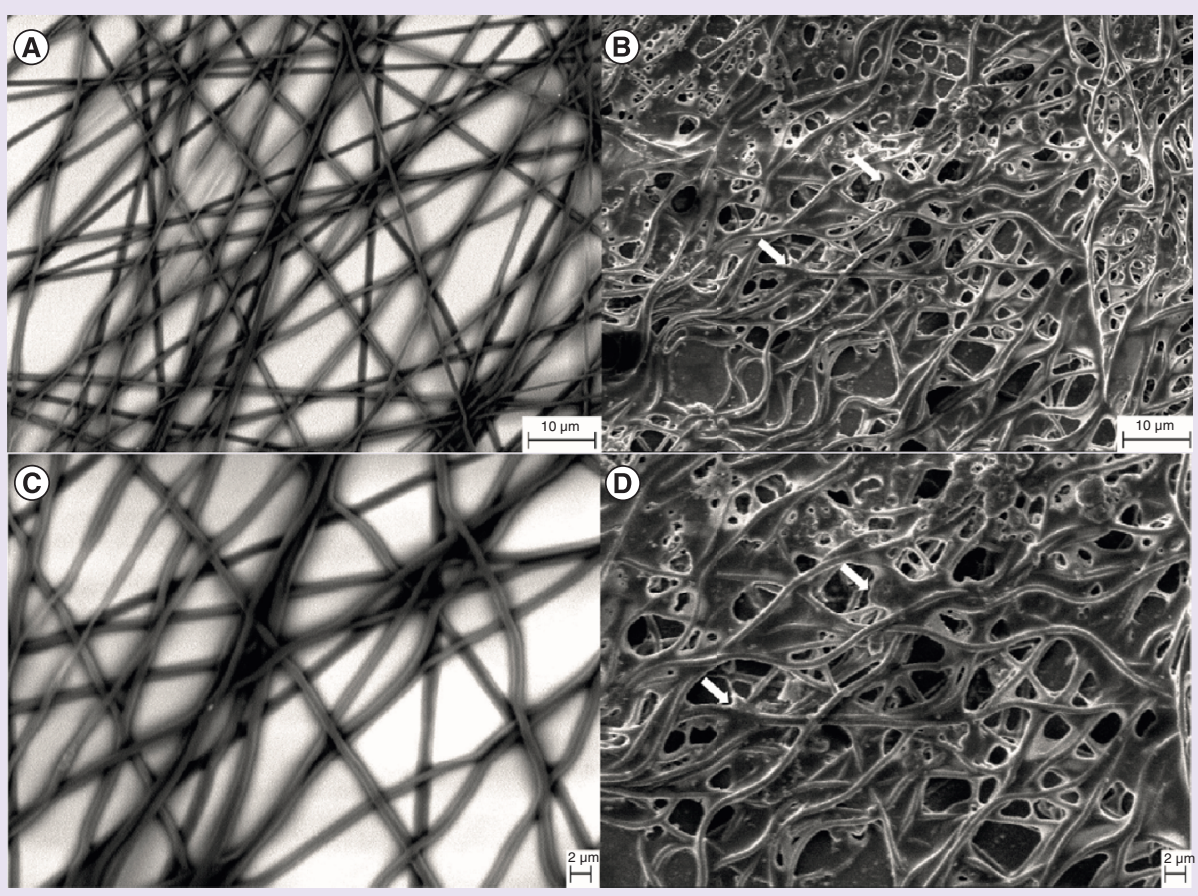

Figure 2. Scanning electron microscopy images of silk fibroin nanofiber scaffold. (A \& C) and cell attached to the silk scaffold (B \& D). The arrow denotes the adhered cells. Scale bar: (A \& B) $10 \mu \mathrm{m}$, (C) and (D) $2 \mu \mathrm{m}$.

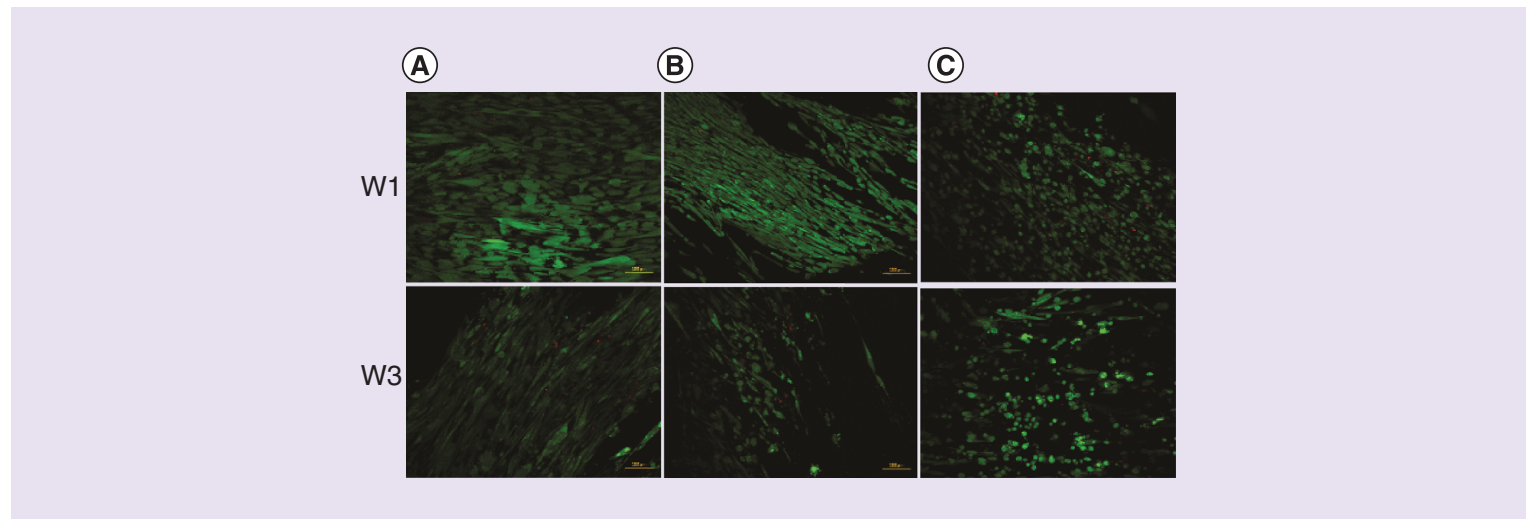

Figure 3. Live dead assay of cells cultured on silk fibroin nanofiber scaffolds at weeks 1 and 3. (A) Mesenchymal stem cells monoculture; (B) induced endothelial cells-mesenchymal stem cells co-culture and (C) endothelial cells-mesenchymal stem cells co-culture. Scale bar: $100 \mu \mathrm{m}$.

\section{Silk fibroin nanofiber scaffold}

Deposition of electrospun SF nanofibers onto the target was achieved by electrospinning at $14 \mathrm{kV}$. Further, SEM analysis of SF nanofibers revealed the smooth surface and the nanofibers had diameters in the range of 400-500 nm (Figure 2). Further, morphological analysis of the nanofiber scaffold exhibited the homogeneous deposition of the fibers covering the total surface area of target (Figure 2). Furthermore, SEM images (Figure 2) of the scaffolds with and without cells clearly showed the cell attachment to the silk nanofiber of the scaffold. The cellular process and spreadings of the attached cells were evident from the images.

Live/dead assay

The fluorescent microscopic pictures (Figure 3) of the live/dead assay showed excellent viability for all three groups 


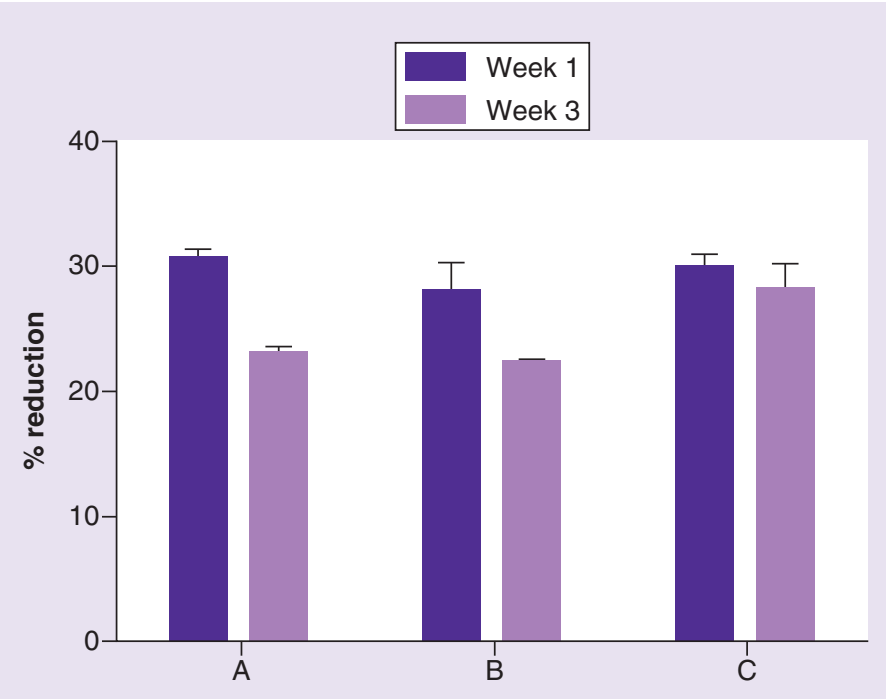

Figure 4. AlamarBlue assay of cells cultured on silk fibroin nanofiber scaffolds at weeks 1 and 3. (A) Mesenchymal stem cells monoculture; (B) induced endothelial cell-mesenchymal stem cells co-culture and (C) endothelial cell-mesenchymal stem cell co-culture.

without substantial dead cells. The cells attached well to the SFNS in all the groups, indicating the good structural support provided by silk materials (Figure 2).

\section{AlamarBlue assay}

The AlamarBlue dye reduction is indicative of cell proliferation. The dye reduction levels correlate positively with cell proliferation. The AlamarBlue reduction test showed good levels of cell proliferation for all the groups (Figure 4). No significant difference was observed between the groups.

\section{Differentiation studies}

ALP assay \& staining

The measured ALP activity was normalized to the total protein content. Compared with the control group A, group B showed significantly higher $(\mathrm{p}<0.05)$ ALP values at week 3 (Figure 5A). Also, group C $(\mathrm{p}<0.05)$ expressed higher ALP levels than group A at week 3. The ALP-stained pictures complied well with the assay results (Figure 5B).

\section{Calcium assay \& staining}

The calcium deposition followed the same pattern as ALP assay (Figure 6A). Group B expressed significantly higher $(\mathrm{p}<0.05)$ calcium levels than the control group $A$ at week 3 . Also, values of group $C$ were significantly higher $(p$ $<0.05)$ than the control group A at week 3. Scaffolds stained with Alizarin Red corroborated with the results of calcium assay (Figure 6B).

\section{Gene studies}

RT-qPCR analysis (at week 3) confirmed the differentiation and expression of the osteogenic marker molecule $O C$ and angiogenic marker molecule $A N G P T 1$ in the co-culture groups (Figure 7). The co-culture groups showed a strong expression of $O C$ in comparison to the control group A. The induced co-culture group B showed higher $O C$ expression than MSC monoculture. The expression of $A N G P T 1$ was observed to be higher in groups $\mathrm{B}$ and $\mathrm{C}$ as compared with the monoculture group A.

\section{Discussion}

Resulting data have shown that iECs can play the role of ECs in a co-culture with UCMSCs on a silk nanofiber scaffold. It is also evident that the osteodifferentiation of MSC $-\mathrm{iEC}$ co-cultures is far superior to MSC monocultures. 


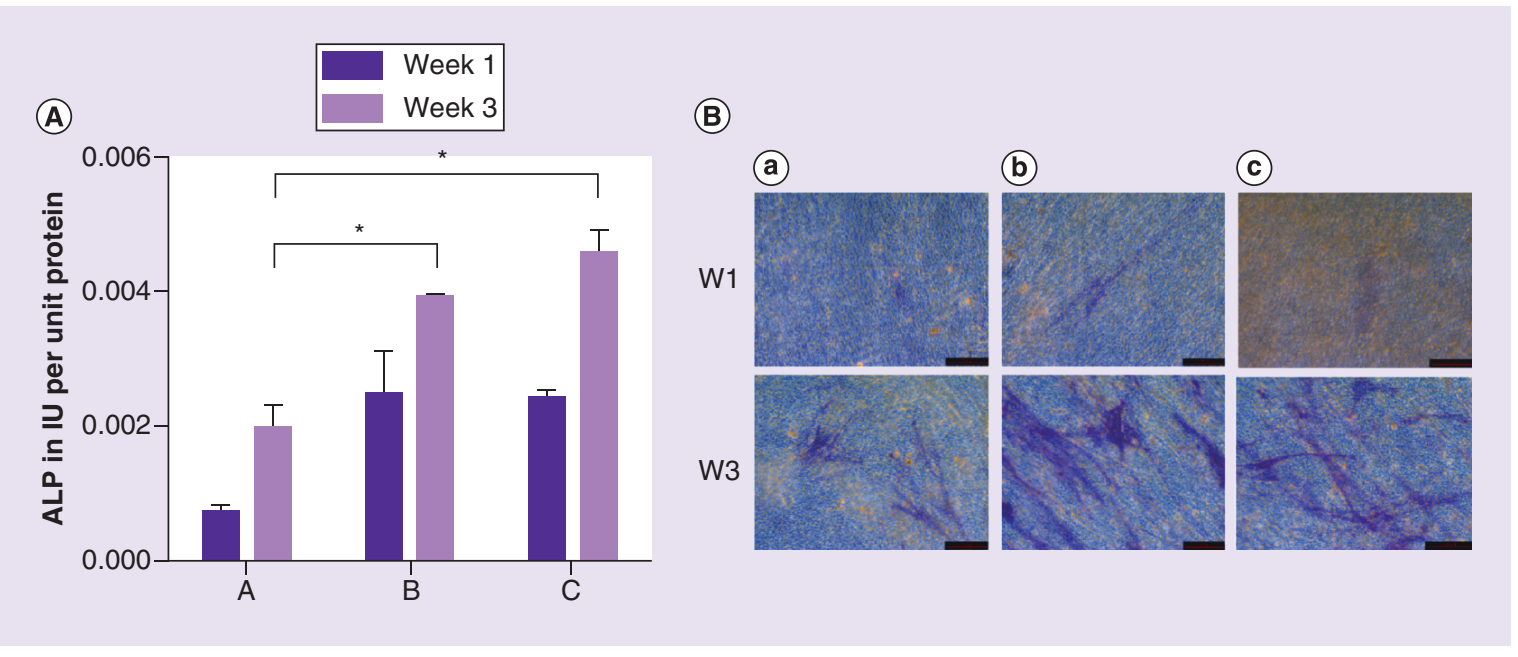

Figure 5. ALP assay and staining. (A) ALP assay of cells cultured on silk fibroin nanofiber scaffolds at weeks 1 and 3 . The differences are considered significant at ${ }^{*} p<0.05$. (B) ALP staining of cells cultured on silk fibroin nanofiber scaffolds at weeks 1 and 3. (A) Mesenchymal stem cell monoculture; (B) induced endothelial cells-mesenchymal stem cells co-culture and $(C)$ endothelial cells-mesenchymal stem cells co-culture.

Scale bar: $100 \mu \mathrm{m}$.

(A)

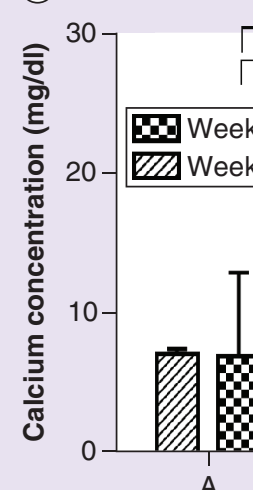

A
*
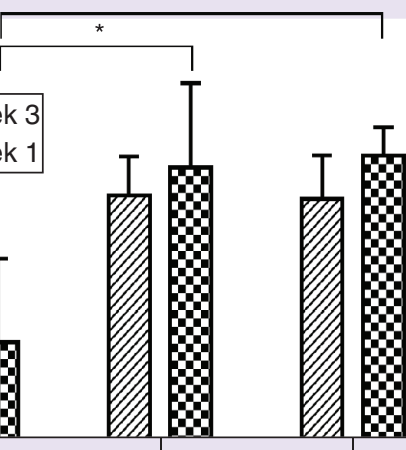

$\mathrm{B}$

C

(B)

(A)

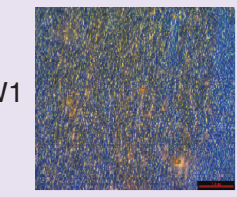

W3

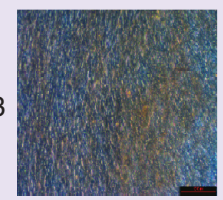

(B)

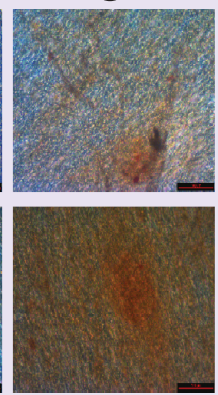

(C)

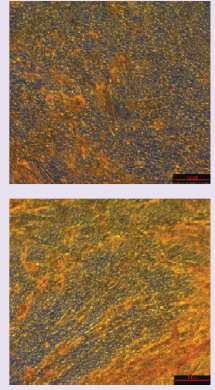

Figure 6. Calcium assay and staining. (A) Calcium assay of cells cultured on silk fibroin nanofiber scaffolds at weeks 1 and 3. The differences are considered significant at $* p<0.05$. (B) Alizarin Red staining of cells cultured on silk fibroin nanofiber scaffolds at weeks 1 and 3. (A) Mesenchymal stem cell monoculture; (B) induced endothelial cells-esenchymal stem cells co-culture and (C) endothelial cells-mesenchymal stem cells co-culture. Scale bar: $100 \mu \mathrm{m}$. MSC: Mesenchymal stem cell.

In this study, we have used two different co-culture groups to understand the role of endothelial differentiation of MSCs in overall osteodifferentiation in a silk-based scaffold when in a co-culture model.

Bone tissue engineering, based on stem cells and biomaterials, may provide a chance of reconstructing the lost bone tissue. These cells should be committed to the osteogenic lineage for the implant to be able to fill bone defects. It is established that MSCs play a vital role in bone tissue regeneration and can create a regenerative microenvironment [30]. Because of their differentiation capacity, MSCs prove to be promising candidates for tissueengineering applications. UCMSCs were found to be viable, and they were not rejected even after 4 months of transplantation as xenografts, suggesting these cells to be a promising cell source for transplantation [26,31].

The notion of using co-culture of MSCs with ECs seems to be promising for bone-regeneration applications [5]. The MSC-EC interaction may have a profound effect on proliferation, differentiation, cell fate and consequent angiogenesis and osteogenesis [6]. Direct contact of human ECs and MSCs has shown to increase the mineralization 

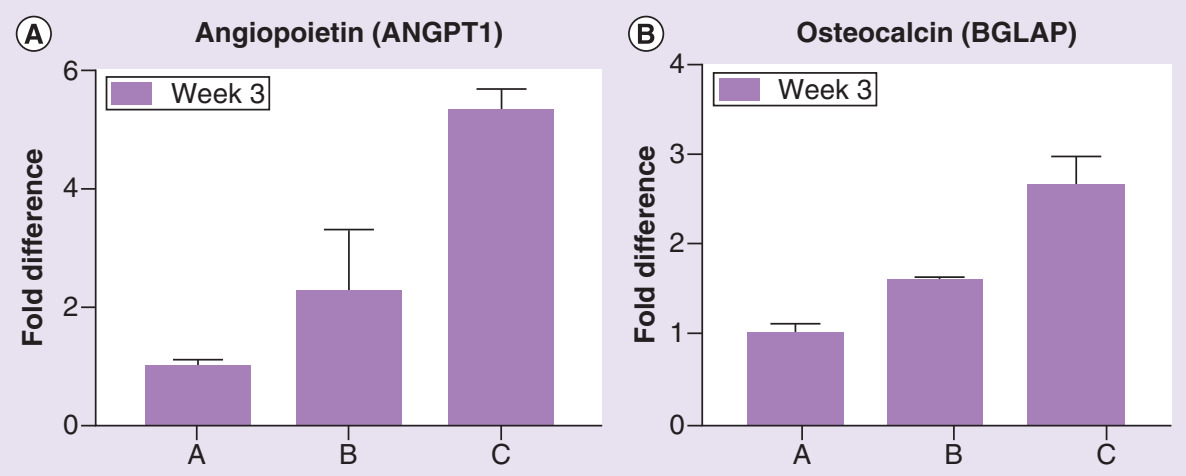

Figure 7. Real time PCR analysis of cells cultured on silk fibroin nanofiber scaffolds at week $\mathbf{3}$ for Angiopoietin 1 and osteocalcin. (A) Mesenchymal stem cell monoculture; (B) induced endothelial cells-mesenchymal stem cell co-culture and (C) endothelial cells-mesenchymal stem cell co-culture.

and ALP activity (an early osteogenic marker) both in vitro and in vivo [32-35]. In addition, Sun et al. reported that MSC-EC co-culture improved bone formation and blood perfusion of the transplanted constructs [11]. Gershovich et al. established that co-culturing osteogenically precultured MSCs with HUVECs in a 1:1 ratioenhanced osteogenic differentiation compared with MSC cultures alone. They showed that the ALP levels in co-cultures were higher than that of monocultures. They also observed the higher production of collagen and glycosaminoglycans [12]. This accentuates the role of ECs as osteogenic mediators in co-culture systems.

Most of the times, ECs are isolated from large blood vessels, which are then seeded, in a suitable scaffold. Although, these ECs have several drawbacks, including low proliferation rate in culture, lack of availability of large vessels and the tendency to de-differentiate in culture after three passages $[18,27,36]$. Previous co-culture studies used MSCs and ECs obtained from two different sources or even species, which restricted potential clinical applications [20,37]. Endothelial progenitor cells, which can differentiate into mature ECs both in vitro and in vivo, have also been employed as EC source [38]. However, these cells have the disadvantage of limited proliferation capacity and losing their characteristics after prolonged expansion [39,40]. Hence, there is a need for an alternative source of ECs.

The cells isolated from human umbilical cord are indicative of mesoangioblasts in phenotype, which were mesenchymal-like cells primarily related to the walls of large vessels. They were found to differentiate into both mesodermal and endothelial lineages, and also expressed EC markers [41,42]. Another explanation is that pluripotent MSCs were found in early phase mesoderm and ectoderm, making it possible for them to differentiate into mesoderm and ectoderm lineages. Since ECs belong to mesoderm lineage, it is safe to say that MSCs can transdifferentiate into endothelial lineage [43].

Endothelial differentiation of MSCs has been reported earlier by many groups [12,18,19,44,45]. Alaminos et al. showed that endothelial differentiation of MSCs resulted in expression of high levels of VEGFR1, CD36 and endothelin converting enzyme 2 that were similar to that of HUVECs. They reported that the receptor profile of the differentiated MSCs matched HUVECs and was clearly different from the MSC profile [23]. MSCs subjected to endothelial differentiation functioned similarly to HUVECs in Matrigel sprouting assay [13]. Tao et al. also showed that endothelial differentiation of MSCs leads to the similar expression of von Willebrand factor (vWF) and CD 31 as that of HUVECs [20].

Endothelial differentiation of MSCs was achieved by growing MSCs in EC growth medium for few days. The time of induction varied from one week to two weeks. Some carried out the endothelial differentiation of MSCs for seven days [21], while some for ten days [13], and others for 12 days [10], and 14 days [20]. The initial cell density of seeded MSCs also differed among studies from 3000 to $5000 \mathrm{cells} / \mathrm{cm}^{2}$. While Portalska et al. used 3000 cells $/ \mathrm{cm}^{2}$ [13] Chen's group used $5000 \mathrm{cells} / \mathrm{cm}^{2}$ [10]. Most of the studies used EGM-2 for endothelial induction [12,19], but few others used a basal medium with VEGF [21] and also including other factors such as epidermal growth factor and hydrocortisone with VEGF [10].

Another significant factor is the source of MSCs, which were differentiated into endothelial phenotype. Most of the studies used BM-MSCs for endothelial differentiation $[12,19,30,44]$. Although, it was reported that UCMSCs 
were more favorable for neovascularization of engineered tissues than BM-MSCs [10,46]. UCMSCs expressed higher levels of endothelial-specific markers such as von Willebrand factor, Flk-1 and VE-cadherin than BM-MSCs after endothelial induction. Also, UCMSCs had higher tubule diameter, length and area than BM-MSCs following endothelial differentiation.

Tao et al. have reported the use of iECs in co-culture with MSCs [20]. Although they found the ALP activity of MSC/iEC co-cultures to be higher than MSC monocultures, the study did not dwell into any more detailed assays or gene expression studies. Also, they used MSCs from bone marrow for their work.

The role of iECs in co-culture with MSCs was also reported by Tao and group. They observed that the activity of BM-MSC/iEC co-culture group was significantly higher than BM-MSCs alone. However, they did not perform any comparison of iEC co-cultures with EC co-cultures. Most of the previous studies have tested the endothelial differentiation of MSCs under 2D culture conditions $[17,18,30,45]$. Very few groups have studied this endothelial differentiation on $\beta$-tricalcium phosphate and poly-L-lactide/poly(lactic-co-glycolic acid) scaffolds [12,19]. But these studies do not dwell on the role of iECs in co-culture in detail. We have reported the comparison of co-cultures regarding endothelial differentiation of MSCs with the traditional MSC co-cultures and monoculture, which is unique. It has been observed that MSCs interact and attract to in vitro formed vascular structures [47].

Lozito et al. observed the tendency of MSCs to differentiate toward endothelial and smooth muscle lineages upon changing the matrix composition and crosslinking level [48]. It was also reported that MSCs cultured on Matrigel, a basement membrane matrix showed higher expression of $K D R$, which stimulated them to differentiate into endothelial lineage [13]. Thus, there is a great need for appropriate structural scaffold and scalable source of ECs supporting the osteogenic induction of MSCs. Various fabrication techniques have been employed to reconstruct the natural extracellular matrix (ECM) of bone tissue. Especially, 3D scaffolds constructed by electrospinning serve as promising bone tissue-engineering scaffolds due to their simulation of natural ECM structures, high surface area and porosity $[24,40,49]$. To address some of these problems, we have fabricated silk fibroin nanofibrous scaffold and studied its ability for supporting the osteogenic differentiation of MSC-ECs co-cultures.

Silk fibroin is a widely known natural biomaterial acquired from B. mori cocoons and contains alanine, glycine and serine $[42,43]$. Previous reports have shown that silk fibroin has excellent biocompatibility, strong mechanical properties and low degradation rate [50-52]. BM-MSCs produced new ECM on patterned silk fibroin surfaces [53]. Silk fibroin supported BM-MSC adhesion and proliferation required for bone regeneration [54]. Electrospun 3D silk fibroin scaffolds enhanced the preosteoblast proliferation and ALP activity of the osteoblasts [55,56].

In this study, we have used one single MSC source to get MSC-EC co-culture and compared it with the two-source MSC-EC co-culture. iECs were produced and their functional capacity was assessed for inducing the osteogenic differentiation of MSCs within the SFNS. Our study differs from the previously published work due to the types of cells used, methodology and application. For instance, Alaminos et al. transdifferentiated Wharton's jelly stem cells to endothelial-like stem cells for use in vascular tissue engineering [23]. Portalska’s group established a protocol for endothelial differentiation of human mesenchymal stromal cells [13]. The aim of our study is based on the use of autologous cells for two different cell types, which varies differently from the other studies wherein their major concern is to address the vascular tissue-engineering applications [57,58].

FDA/PI staining showed satisfactory live cell attachment on 2D culture plate as well as the 3D silk nanofiber scaffolds. This was observed in both MSC monoculture and co-culture groups at weeks 1 and 3 time points. Silk nanofiber scaffolds served as a good support for the cell attachment. The proliferation rate of both MSC monoculture and co-culture was higher on 2D culture plate as compared with the electrospun silk fibroin scaffolds at weeks 1 and 3. Since the process of MSC differentiation marks a dip in cell proliferation [59], it is deduced that MSC differentiation is higher on silk scaffolds than the 2D plate. Also, higher osteogenic differentiation as seen by ALP staining, was higher on 3D silk scaffolds. Hence, silk nanofiber scaffolds were seen to be better for cell proliferation and osteogenic differentiation as compared with the 2D culture plate (Supplementary Figure 1-3).

In the present study, iECs obtained from endothelial induction of UCMSCs showed tube-like formation in Matrigel and stained positively for lectin. The tube formation in the Matrigel and the positive expression of lectin are characteristic attributes of ECs. The MSCs were successfully characterized by the trilineage differentiation of the UCMSCs, combined with the positive expression of the MSC markers. The attachment of cells on the silk nanofiber scaffolds was confirmed by the SEM images. The cell viability was seen from the images of FDA/PI assay. The green-labeled viable cells were clearly evident in all the groups. The AlamarBlue results showed that the cells of all groups proliferated well. The dye reduction of the groups is indicative of their proliferation. Hence, it is deduced that the cells of all groups possessed good proliferation capacity. The SFNS consisted of a cover glass of $13 \mathrm{~mm}$ 
diameter upon which the silk fibroin nanofibers were deposited. Due to the limited space on the scaffold and the long duration of 3 weeks, the cells might have been over confluent, which has been shown to cause cell death [60]. Although, the cells viability seems to be lower at week 3, good cell proliferation was seen in the AlamarBlue assay at week 3. Also, the supplementary results of live-dead assay prove that the silk nanofiber scaffold serves as good support for the cell attachment.

The ALP activity clearly showed the osteogenic differentiation to be higher for iEC-based co-culture group as compared with the monoculture group A and was proximal to the EC-based co-culture group. Both the ALP assay and ALP staining showed the same trend. The calcium assay and the calcium staining showed that the calcium levels were higher for iEC-based co-culture group as compared with the MSC monoculture group A and were proximal to the EC-based co-culture group. The 3D scaffolds used for the study were thin and porous 3D nanofibrous scaffolds. Hence, the light was partly permeable and the seeded cells could be imaged and the deposited calcium could be stained as mentioned in the methods section without any problem. Hence, just the incorporation of iECs into MSC culture resulted in higher osteogenic differentiation. Since iECs are obtained from UCMSCs, one single source of MSCs can be used to get two different kinds of cells, which can be co-cultured for the achievement of enhanced osteodifferentiation. This would help surpass the tedious work of obtaining two different kinds of cells using different isolation protocols with difficult ethical approval for two cell source samples. Since it requires just 12 days of endothelial induction of UCMSCs, UCMSC/iEC co-culture is an easy and superior choice over the conventional MSC/EC co-culture. This proves the role of iECs in co-culture with MSCs to induce osteodifferentiation.

Meinel et al. reported enhanced bone formation potential when MSCs were predifferentiated toward osteoblastic lineage [8]. Binder and group also reported increased cell survival when cells were precultured in osteogenic medium [9]. As few such reports suggested the predifferentiation of MSCs for better bone formation [61,62], we added a predifferentiated MSC co-culture group in the study. However, there was no significant difference of osteogenic differentiation seen between predifferentiated MSC co-culture group and nonpredifferentiated MSC (data not shown). Therefore, it was deduced that osteogenic differentiation of MSCs before co-culture does not have any effect on their metabolic activity, which has been reported earlier [63,64]. This shows that osteogenic preinduction of MSCs before co-culture did not help in increase of osteogenic differentiation.

To establish that iECs can act as a substitute for ECs, we checked the expression of the angiogenic marker, Angiopoietin-1, in all the co-culture groups [65,66]. Angiopoietin-1 acts as a chemoattractant for ECs during angiogenesis, and also EC sprouting [67]. The results positively demonstrate that the iECs highly express angiogenic markers in co-culture with MSCs. Osteocalcin (also known as BGLAP) is a middle-to-late osteogenic marker, mainly expressed by late osteoblasts and osteocytes [68]. It plays a significant role in mineralization of the bone tissue [69]. The $O C$ gene expression trend in the co-culture groups is supported by the observations made in the Alizarin Red staining for calcium deposition. Although the iEC-based group: group B shows lower gene expression as compared with the EC-based groups: group C, its expression is still higher than the monoculture control group A. Hence, it is noteworthy that the usage of one cell source for co-culture can increase the osteogenic and endothelial gene expression as compared with its application as a monoculture.

Our study shows the intelligent use of one source of MSCs to obtain two cell types, which on co-culture, attain higher osteogenic differentiation than culturing MSCs alone. In addition to the previous findings, we have also proved that iECs play the role of ECs when co-cultured with MSCs. Osteogenic preinduction of MSCs before co-culture did not favor osteogenic differentiation. In the future, autologous MSCs for the development of cocultures would be of great help for use in personalized medicine. One type of cell, that is, MSC from the patient can give rise to iECs, which can be co-cultured with primarily obtained MSCs to form bone. This method of bone formation can be further translated into various new technologies for bone tissue-engineering applications.

\section{Conclusion}

We have compared the osteogenic differentiation of isogenic iEC-MSC co-cultures to that of UCMSC monoculture and EC-MSC co-culture. We found that iEC-based co-culture showed similar osteogenic differentiation to that of EC-MSC-based co-culture. This proved that iECs are mimicking ECs when co-cultured with MSCs. We show that one MSC source can be used to give rise to both MSCs and iECs. Thus, isogenic MSCs and iECs on SFNS hold potential for bone tissue engineering (Figure 8). 


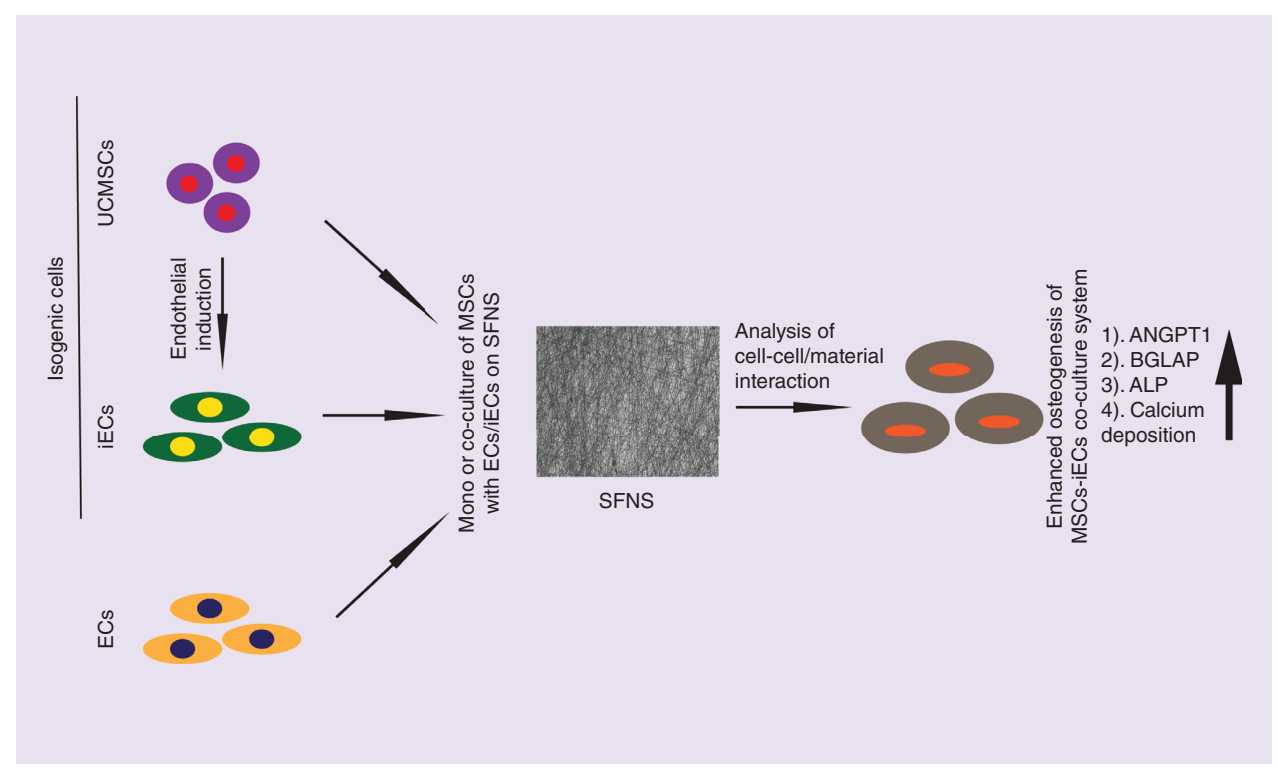

Figure 8. Isogenic induced-endothelial cells enhance osteogenic differentiation of mesenchymal stem cells on silk fibroin scaffold.

EC: Endothelial cell; iEC: Induced endothelial cell; SFNS: Silk fibroin nanofiber scaffold; UCMSC: Umbilical cord mesenchymal stem cells.

\section{Translational perspective}

Future directions toward the successful development of autologous cells for personalized medicine would be a great milestone to reach. MSCs procured from the patient can be used to create iECs, which when co-cultured with MSCs have the ability to form a bone-like matrix. Sophisticated methods like 3D bioprinting can also be used to form bone that can then be transplanted back into the patient avoiding any problems of tissue rejection or ethical issues. Owing to the multiple cell types, matrix components and their organization, it becomes difficult to grow a living tissue or organ in their native state. Besides being used to replace damaged organs, the 3D constructs produced by tissue engineering can also be used as experimental platforms. The $3 \mathrm{D}$ bioprinting is a promising approach for tissue regeneration and remains a transformative tool in several fields. With advanced technology and increased accessibility, the process should be well improved to develop fully functional, ready-to-implant constructs in the coming years.

Supplementary data

To view the supplementary data that accompany this paper please visit the journal website at: www.futuremedicine.com/doi/full/ 10.2217/rme-2018-0166

\section{Acknowledgments}

The authors would like to thank $V$ Swarnalatha for assistance with scanning electron microscopy images.

\section{Financial \& competing interest disclosure}

The authors have no relevant affiliations or financial involvement with any organization or entity with a financial interest in or financial conflict with the subject matter or materials discussed in the manuscript. This includes employment, consultancies, honoraria, stock ownership or options, expert testimony, grants or patents received or pending, or royalties.

No writing assistance was utilized in the production of this manuscript.

\section{Ethical conduct of research}

All procedures performed in studies involving human participants were in accordance with the ethical standards of the Institutional Ethics Committee and Institutional Committee for Stem Cell Research of IT Hyderabad and with the 1964 Helsinki declaration and its later amendments or comparable ethical standards. In addition, for investigations involving human subjects, informed consent has been obtained from the participants involved. 


\section{Open access}

This work is licensed under the Attribution-NonCommercial-NoDerivatives 4.0 Unported License. To view a copy of this license, visit http://creativecommons.org/licenses/by-nc-nd/4.0/

\section{Summary points}

- Direct interaction of endothelial cells (ECs) with mesenchymal stem cells (MSCs) results in the osteogenic differentiation of MSCs in vitro and in vivo. Induced ECs (iECs) were produced by endothelial differentiation of MSCs and co-cultured with MSCs on silk fibroin nanofiber scaffolds (SFNS).

- Previous studies did not dwell into any detailed assays or gene expression studies. Also, they used MSCs from bone marrow for their work and did not perform any comparison of iEC co-cultures with EC co-cultures.

- In this study, we investigated the role of iECs in MSCs/iECs Co-culture and assessed their osteogenic ability in comparison with MSCs monoculture and MSCS/ECs co-culture on SFNS. We have reported the comparison of co-cultures regarding endothelial and osteogenic differentiation of MSCs, which is unique.

- ALP levels and calcium deposition of the co-cultures containing iECs were found to be higher than the monoculture MSCs group and proximal to the co-cultures containing ECs. Furthermore, it is noteworthy that the usage of isogenic iECs for co-culture can increase the osteogenic and endothelial gene expression.

- These findings suggest that iECs mimic ECs when co-cultured with MSCs and exert their osteogenic potential. Thus, we show that one MSCs source can be used to give rise to both MSCs and iECs for osteogenic interactions. The resulting isogenic MSCs/iECs co-culture system provides a new option for bone tissue-engineering applications, and SFNS holds potential as a supporting scaffold.

\section{References}

1. Zuk PA, Zhu M, Mizuno H et al. Multilineage cells from human adipose tissue: implications for cell-based therapies. Tissue Eng. 7(2), 211-228 (2001).

2. Kita K, Gauglitz GG, Phan TT et al. Isolation and characterization of mesenchymal stem cells from the sub-amniotic human umbilical cord lining membrane. Stem Cells Dev. 19(4), 491-502 (2010).

3. Maslova OO, Shuvalova NS, Sukhorada OM et al. Heterogeneity of umbilical cords as a source for mesenchymal stem cells. Dataset Pap. Biol. 2013, 1-4 (2013).

4. Kaigler D, Krebsbach PH, West ER et al. Endothelial cell modulation of bone marrow stromal cell osteogenic potential. FASEB J. 19(6), 665-667 (2005).

5. Shahdadfar A, Frønsdal K, Haug T et al. In vitro expansion of human mesenchymal stem cells: choice of serum is a determinant of cell proliferation, differentiation, gene expression, and transcriptome stability. Stem Cells 23(9), 1357-1366 (2005).

6. Ball SG, Shuttleworth AC, Kielty CM. Direct cell contact influences bone marrow mesenchymal stem cell fate. Int. J. Biochem. Cell Biol. 36(4), 714-727 (2004).

7. Fu Y-S, Cheng Y-C, Lin M-YA et al. Conversion of human umbilical cord mesenchymal stem cells in Wharton's jelly to dopaminergic neurons in vitro: potential therapeutic application for Parkinsonism. Stem Cells 24(1), 115-124 (2006).

8. Meinel L, Betz O, Fajardo R et al. Silk based biomaterials to heal critical sized femur defects. Bone 39(4), 922-931 (2006).

9. Binder BY, Genetos DC, Leach JK. Lysophosphatidic acid protects human mesenchymal stromal cells from differentiation-dependent vulnerability to apoptosis. Tissue Eng. Part A 20(7-8), 1156-1164 (2014).

10. Chen M-Y, Lie P-C, Li Z-L et al. Endothelial differentiation of Wharton's jelly-derived mesenchymal stem cells in comparison with bone marrow-derived mesenchymal stem cells. Exp. Hematol. 37(5), 629-640 (2009).

11. Sun $\mathrm{H}, \mathrm{Qu} \mathrm{Z}$, Guo Y et al. In vitro and in vivo effects of rat kidney vascular endothelial cells on osteogenesis of rat bone marrow mesenchymal stem cells growing on polylactide-glycoli acid (PLGA) scaffolds. Biomed. Eng. OnLine 6(1), 41 (2007).

12. Gershovich JG, Dahlin RL, Kasper FK et al. Enhanced osteogenesis in cocultures with human mesenchymal stem cells and endothelial cells on polymeric microfiber scaffolds. Tissue Eng. Part A 19(23-24), 2565-2576 (2013).

13. Janeczek Portalska K, Leferink A, Groen N et al. Endothelial differentiation of mesenchymal stromal cells. PLoS ONE 7(10), e46842 (2012).

14. Rath SN, Strobel LA, Arkudas A et al. Osteoinduction and survival of osteoblasts and bone-marrow stromal cells in 3D biphasic calcium phosphate scaffolds under static and dynamic culture conditions. J. Cell Mol. Med. 16(10), 2350-2361 (2012).

15. Rath SN, Nooeaid P, Arkudas A et al. Adipose- and bone marrow-derived mesenchymal stem cells display different osteogenic differentiation patterns in 3D bioactive glass-based scaffolds: ADSCs and BMSCs show different osteogenic differentiation patterns in 3D bioactive glass-based scaffolds. J. Tissue Eng. Regen. Med. 10(10), E497-E509 (2013). 
16. La Rocca G, Lo Iacono M, Corsello T et al. Human Wharton's jelly mesenchymal stem cells maintain the expression of key immunomodulatory molecules when subjected to osteogenic, adipogenic and chondrogenic differentiation in vitro: new perspectives for cellular therapy. Curr. Stem Cell Res. Ther. 8(1), 100-113 (2013).

17. Ciombor DMCK, Aaron RK. The role of electrical stimulation in bone repair. Foot Ankle Clin. 10(4), 579-593 (2005).

18. Sarraf CE, Otto WR, Eastwood M. In vitro mesenchymal stem cell differentiation after mechanical stimulation: mechanical stimulation differentiates MSC. Cell Prolif. 44(1), 99-108 (2011).

19. Carpentier B, Layrolle P, Legallais C. Bioreactors for bone tissue engineering. Int. J. Artif. Organs 34(3), 259-270 (2011).

20. Tao J, Sun Y, Wang Q, Liu C. Induced endothelial cells enhance osteogenesis and vascularization of mesenchymal stem cells. Cells Tissues Organs 190(4), 185-193 (2009).

21. Oswald J, Boxberger S, Jørgensen B et al. Mesenchymal stem cells can be differentiated into endothelial cells in vitro. Stem Cells 22(3), 377-384 (2004).

22. Xu L, Wang S, Sui X et al. Mesenchymal stem cell-seeded regenerated silk fibroin complex matrices for liver regeneration in an animal model of acute liver failure. ACS Appl. Mater. Interfaces 9(17), 14716-14723 (2017).

23. Alaminos M, Pérez-Köhler B, Garzón I et al. Transdifferentiation potentiality of human Wharton's jelly stem cells towards vascular endothelial cells. J. Cell. Physiol.223(3), 640-647 (2010).

24. Jain RK. Molecular regulation of vessel maturation. Nat. Med. 9(6), 685-693 (2003).

25. Damoulis PD, Drakos DE, Gagari E et al. Osteogenic differentiation of human mesenchymal bone marrow cells in silk scaffolds is regulated by nitric oxide. Ann. NY Acad. Sci. 1117(1), 367-376 (2007).

26. Ko E, Lee JS, Kim H et al. Electrospun silk fibroin nanofibrous scaffolds with two-stage hydroxyapatite functionalization for enhancing the osteogenic differentiation of human adipose-derived mesenchymal stem cells. ACS Appl. Mater. Interfaces 10(9), 7614-7625 (2017).

27. Mennan $\mathrm{C}$, Wright $\mathrm{K}$, Bhattacharjee A et al. Isolation and characterisation of mesenchymal stem cells from different regions of the human umbilical cord. BioMed Res. Int. 2013, 1-8 (2013).

28. Rath SN, Brandl A, Hiller D et al. Bioactive copper-doped glass scaffolds can stimulate endothelial cells in co-culture in combination with mesenchymal stem cells. PLoS ONE 9(12), e113319 (2014).

29. Madduri S, Papaloïzos M, Gander B. Trophically and topographically functionalized silk fibroin nerve conduits for guided peripheral nerve regeneration. Biomaterials 31(8), 2323-2334 (2010).

30. Bidarra SJ, Barrias CC, Barbosa MA et al. Phenotypic and proliferative modulation of human mesenchymal stem cells via crosstalk with endothelial cells. Stem Cell Res. 7(3), 186-197 (2011).

31. Yang SY, Hwang TH, Che L et al. Membrane-reinforced three-dimensional electrospun silk fibroin scaffolds for bone tissue engineering. Biomed. Mater. 10(3), 035011 (2015).

32. Ehnert $\mathrm{S}$, van Griensven $\mathrm{M}$, Unger $\mathrm{M}$ et al. Co-culture with human osteoblasts and exposure to extremely low frequency pulsed electromagnetic fields improve osteogenic differentiation of human adipose-derived mesenchymal stem cells. Int. J. Mol. Sci. 19(4), 994 (2018).

33. Kim J, Kim HN, Lim K-T et al. Synergistic effects of nanotopography and co-culture with endothelial cells on osteogenesis of mesenchymal stem cells. Biomaterials 34(30), 7257-7268 (2013).

34. Steward AJ, Cole JH, Ligler FS et al. Mechanical and vascular cues synergistically enhance osteogenesis in human mesenchymal stem cells. Tissue Eng. Part A 22(15-16), 997-1005 (2016).

35. Freeman MFE, Haugh DMG, McNamara DL. An in vitro bone tissue regeneration strategy combining chondrogenic and vascular priming enhances the mineralisation potential of MSCs in vitro whilst also allowing for vessel formation. Tissue Eng. Part A21(7-8), 1320-1332 (2015).

36. Ormiston ML, Toshner MR, Kiskin FN et al. Generation and culture of blood outgrowth endothelial cells from human peripheral blood. J. Vis. Exp. (106), e53384 (2015).

37. Pill K, Hofmann S, Redl $\mathrm{H}$ et al. Vascularization mediated by mesenchymal stem cells from bone marrow and adipose tissue: a comparison. Cell Regen. 4(1), 4:8 (2015).

38. Asahara T, Murohara T, Sullivan A et al. Isolation of putative progenitor endothelial cells for angiogenesis. Science 275(5302), 964-966 (1997).

39. Patel J, Donovan P, Khosrotehrani K. Concise review: functional definition of endothelial progenitor cells: a molecular perspective: defining an EPC. Stem Cells Transl. Med. 5(10), 1302-1306 (2016).

40. Schatteman GC, Dunnwald M, Jiao C. Biology of bone marrow-derived endothelial cell precursors. Am. J. Physiol.-Heart Circ. Physiol. 292(1), H1-H18 (2007).

41. Shafiee A, Patel J, Hutmacher DW et al. Meso-endothelial bipotent progenitors from human placenta display distinct molecular and cellular identity. Stem Cell Rep. 10(3), 890-904 (2018).

42. Crisan M. Transition of mesenchymal stem/stromal cells to endothelial cells. Stem Cell Res Ther. 4, 95 (2013). 
43. Liu D, Zhang X, Li X et al. Differentiation of the human marrow mesenchymal stem cells into vascular endothelium-like cells in vitro. In: 7th Asian-Pacific Conference on Medical and Biological Engineering. Springer, Heidelberg, Germany, 80-83 (2008).

44. Wang C, Li Y, Yang M et al. Efficient differentiation of bone marrow mesenchymal stem cells into endothelial cells in vitro. Eur. J. Vasc. Endovasc. Surg. 55(2), 257-265 (2018).

45. Liu C, Tsai A-L, Li P-C et al. Endothelial differentiation of bone marrow mesenchyme stem cells applicable to hypoxia and increased migration through Akt and NFkB signals. Stem Cell Res. Ther. 8(1), 29 (2017).

46. Li J, Li D, Liu X et al. Human umbilical cord mesenchymal stem cells reduce systemic inflammation and attenuate LPS-induced acute lung injury in rats. J. Inflamm. 9(1), 33 (2012).

47. Sorrell JM, Baber MA, Caplan AI. Influence of adult mesenchymal stem cells on in vitro vascular formation. Tissue Eng. Part A 15(7), 1751-1761 (2009).

48. Lozito TP, Taboas JM, Kuo CK et al. Mesenchymal stem cell modification of endothelial matrix regulates their vascular differentiation. J. Cell. Biochem. 107(4), 706-713 (2009).

49. Jun I, Han H-S, Edwards J et al. Electrospun fibrous scaffolds for tissue engineering: viewpoints on architecture and fabrication. Int. J. Mol. Sci. 19(3), 745 (2018).

50. Zhang L, Liu X, Li G et al. Tailoring degradation rates of silk fibroin scaffolds for tissue engineering: tailoring degradation rates of silk fibroin scaffolds. J. Biomed. Mater. Res. A 107(1), 104-113 (2019).

51. Maisani M, Pezzoli D, Chassande $\mathrm{O}$ et al. Cellularizing hydrogel-based scaffolds to repair bone tissue: how to create a physiologically relevant micro-environment? J. Tissue Eng. 8, 2041731417712073 (2017).

52. Qi Y, Wang $\mathrm{H}$, Wei $\mathrm{K}$ et al. A review of structure construction of silk fibroin biomaterials from single structures to multi-level structures. Int. J. Mol. Sci. 18(3), 237 (2017).

53. Correia C, Bhumiratana S, Yan L-P et al. Development of silk-based scaffolds for tissue engineering of bone from human adipose-derived stem cells. Acta Biomater. 8(7), 2483-2492 (2012).

54. Jin H. Human bone marrow stromal cell responses on electrospun silk fibroin mats. Biomaterials 25(6), 1039-1047 (2004).

55. Wu J, Zheng A, Liu Y et al. Enhanced bone regeneration of the silk fibroin electrospun scaffolds through the modification of the graphene oxide functionalized by BMP-2 peptide. Int. J. Nanomedicine 14, 733-751 (2019).

56. Persson M, Lehenkari PP, Berglin L et al. Osteogenic differentiation of human mesenchymal stem cells in a 3D woven scaffold. Sci. Rep. 8(1), 10457 (2018).

57. Chang WG, Niklason LE. A short discourse on vascular tissue engineering. NPJ Regen. Med. 2(1), 7 (2017).

58. Belair DG, Whisler JA, Valdez J et al. Human vascular tissue models formed from human induced pluripotent stem cell derived endothelial cells. Stem Cell Rev. Rep. 11(3), 511-525 (2015).

59. Ruijtenberg S, van den Heuvel S. Coordinating cell proliferation and differentiation: antagonism between cell cycle regulators and cell type-specific gene expression. Cell Cycle 15(2), 196-212 (2016).

60. Krampe B, Al-Rubeai M. Cell death in mammalian cell culture: molecular mechanisms and cell line engineering strategies. Cytotechnology 62(3), 175-188 (2010).

61. Brennan MA, Renaud A, Guilloton F et al. Inferior in vivo osteogenesis and superior angiogeneis of human adipose-derived stem cells compared with bone marrow-derived stem cells cultured in xeno-free conditions: ectopic bone formation with BM and at stem cells. Stem Cells Transl. Med. 6(12), 2160-2172 (2017).

62. Laschke MW, Schank TE, Scheuer C et al. In vitro osteogenic differentiation of adipose-derived mesenchymal stem cell spheroids impairs their in vivo vascularization capacity inside implanted porous polyurethane scaffolds. Acta Biomater. 10(10), 4226-4235 (2014).

63. Bok J-S, Byun S-H, Park B-W et al. The role of human umbilical vein endothelial cells in osteogenic differentiation of dental follicle-derived stem cells in in vitro co-cultures. Int. J. Med. Sci. 15(11), 1160-1170 (2018).

64. Li T, Liu ZL, Xiao M et al. Impact of bone marrow mesenchymal stem cell immunomodulation on the osteogenic effects of laponite. Stem Cell Res. Ther. 9(1), 100 (2018).

65. van Sleen Y, Sandovici M, Abdulahad WH et al. Markers of angiogenesis and macrophage products for predicting disease course and monitoring vascular inflammation in giant cell arteritis. Rheumatology doi:10.1093/rheumatology/kez034 (2019) (Epub ahead of print).

66. Shih S-C, Robinson GS, Perruzzi CA et al. Molecular profiling of angiogenesis markers. Am. J. Pathol. 161(1), 35-41 (2002).

67. Ucuzian AA, Gassman AA, East AT et al. Molecular mediators of angiogenesis. J. Burn Care Res. 31(1), 158-175 (2010).

68. Bonewald LF. The amazing osteocyte. J. Bone Miner. Res. 26(2), 229-238 (2011).

69. Tsao Y-T, Huang Y-J, Wu H-H et al. Osteocalcin mediates biomineralization during osteogenic maturation in human mesenchymal stromal cells. Int. J. Mol. Sci. 18(1), 159 (2017). 